\title{
Compreensão de estudantes de enfermagem sobre as Redes de Atenção à Saúde
}

\author{
Nursing undergraduates' understanding of Health Care Networks \\ Comprensión de los estudiantes de enfermería sobre las Redes de Atención a la Salud
}

\author{
Thiago Lopes Silva'; Jamila Geri Tomaschewski-Barlem"; Edison Luiz Devos Barlem "II; \\ Raissa Garcia Brum ${ }^{\prime v}$; Bruna Ruoso da Silva Neutzling ${ }^{v}$; Luciano Garcia Lourençãovl
}

\begin{abstract}
RESUMO
Objetivo: analisar a compreensão de estudantes de graduação em enfermagem sobre as Redes de Atenção à Saúde. Método: estudo descritivo e exploratório, qualitativo, realizado com 27 estudantes em Enfermagem de uma universidade federal do sul do Brasil, por meio de entrevistas semiestruturadas realizadas entre agosto e setembro de 2018. Resultados: foi evidenciado o conhecimento dos estudantes em relação ao conceito e objetivo das Redes de Atenção à Saúde, a identificação de fragilidades na comunicação e a falta de conhecimento do usuário como obstáculos na efetivação das mesmas, a dificuldade em ver a atuação do enfermeiro dentro dos variados serviços das redes e a percepção sobre a fragmentação do processo de formação. Conclusão: o estudo contribui para a discussão sobre a inclusão dos estudantes nas Redes de Atenção à Saúde, com intuito de superar as exigências educacionais que buscam favorecer a efetivação do Sistema Único de Saúde e das Redes de Atenção à Saúde.
\end{abstract}

Descritores: Sistemas de Saúde; Sistema Único de Saúde; Estudantes de Enfermagem; Educação em Enfermagem.

\section{ABSTRACT}

Objective: to examine undergraduate nursing students' understanding of Health Care Networks (HCNs). Method: in this exploratory, qualitative descriptive study, semi-structured interviews were conducted with 27 Nursing students at a federal University in southern Brazil between August and September 2018. Results: the interviews revealed that the students' knowledge related to the $\mathrm{HCNs}^{\prime}$ concept and purpose, beyond the communication weaknesses and users' lack knowledge as obstacles to effective $\mathrm{HCNs}$, the difficulty in seeing nurses' roles in the various network services, and perceived fragmentation in the training process. Conclusion: the study contributed to discussion of inclusion of students in HCRs, with a view to meeting the educational requirements designed to favor implementation of the national health system (SUS) and the HCNs.

Descriptors: Health Systems; Unified Health System; Students, Nursing; Education, Nursing.

\section{RESUMEN}

Objetivo: analizar la comprensión de los estudiantes de enfermería de pregrado sobre las redes de atención de la salud (HCN). Método: en este estudio exploratorio, cualitativo descriptivo, se realizaron entrevistas semiestructuradas a 27 estudiantes de Enfermería de una Universidad federal del sur de Brasil entre agosto y septiembre de 2018. Resultados: las entrevistas revelaron que los conocimientos de los estudiantes relacionados con el concepto y propósito, más allá de las debilidades de comunicación y la falta de conocimiento de los usuarios como obstáculos para una $\mathrm{HCN}$ eficaz, la dificultad para ver el rol de las enfermeras en los distintos servicios de la red y la fragmentación percibida en el proceso de formación. Conclusión: el estudio contribuyó a la discusión de la inclusión de los estudiantes en las HCR, con miras a cumplir con los requisitos educativos diseñados para favorecer la implementación del Sistema Nacional de Salud (SUS) y las HCN..

Descriptores: Sistemas de Salud; Sistema Único de Salud; Estudiantes de Enfermería; Educación en Enfermería.

\section{INTRODUÇÃO}

Os sistemas de atenção à saúde abrangem atividades desenvolvidas por distintas instituições, tendo como propósito principal a promoção, a manutenção e a restauração da saúde de uma população. Estes sistemas buscam responder as demandas e as prioridades apresentadas pela sociedade, considerando suas necessidades de saúde para ofertar seus serviços ${ }^{1}$.

'Enfermeiro. Mestrando em Enfermagem. Membro do Laboratório de Pesquisa, Tecnologia e Inovação na Saúde da Criança e do Adolescente. Universidade Federal de Santa Catarina. Florianópolis, Brasil. E-mail: thiagoslopes@outlook.com. ORCID: https://orcid.org/0000-0002-6310-5825

"Enfermeira. Doutora em Enfermagem. Professora Adjunta. Universidade Federal do Rio Grande. Brasil. E-mail: jamila_tomaschewski@hotmail.com. ORCID: https://orcid.org/0000-0001-9125-9103

I'Enfermeiro. Doutor em Enfermagem. Professor Associado. Universidade Federal do Rio Grande. Brasil. E-mail: ebarlem@gmail.com. ORCID: https://orcid.org/0000-0001-6239-8657 IVEnfermeira. Mestranda em Enfermagem. Membro do Núcleo de Estudos e Pesquisas em Saúde. Universidade Federal do Rio Grande. Brasil. E-mail: raissagbrum@gmail.com. ORCID: https://orcid.org/0000-0002-0120-801X

vEnfermeira. Doutoranda em Enfermagem. Universidade Federal do Rio Grande, Brasil. E-mail: brunaneutzling10@gmail.com. ORCID: https://orcid.org/0000-0002-1964-264X V'Enfermeiro. Doutor em Ciências da Saúde. Professor Titular-Livre. Universidade Federal do Rio Grande. Brasil. E-mail: lucianolourencao.enf@gmail.com. ORCID: https://orcid.org/0000-0002-1240-4702 
No Brasil, a fragmentação do sistema de saúde manifesta-se de distintas formas, ocasionadas pela ausência de coordenação dos serviços, multiplicidade de estruturas de mesmo caráter e ações assistenciais, pelo antagonismo e falta de coerência entre os serviços prestados, pelo desenvolvimento de práticas em locais inapropriados, pela dificuldade de acesso e descontinuidade da assistência, dentre outros aspectos. Estas características acarretam a prestação de serviços de baixa qualidade técnica e humanística e de modo não resolutivo, levando ao uso irracional e ineficiente dos recursos disponíveis no sistema de saúde ${ }^{2}$.

A fragmentação do sistema de saúde associada à transição demográfica e epidemiológica brasileira, com aumento das doenças infecciosas, crônicas e causas externas, torna-se dificultadora da garantia de saúde integral, universal e equitativo aos usuários do Sistema Único de Saúde (SUS) ${ }^{1}$.

Considerando as dificuldades decorrentes da fragmentação dos serviços de saúde que compõe o SUS e buscando superar esta problemática, o Ministério da Saúde publicou, no ano de 2010, a Portaria 4.279, que estabelece as diretrizes para a organização das Redes de Atenção à Saúde (RAS). Destacadas pela Organização Pan-Americana da Saúde como o princípio fundamental de superar a fragmentação do cuidado e enfrentar as necessidades da população ${ }^{3}$, as RAS configuram-se por meio de arranjos capazes de contribuir assertivamente para a assistência contínua, integral e de qualidade à população ${ }^{1}$.

Todavia, a operacionalização das RAS configura-se como desafio aos profissionais que atuam no SUS. O enfermeiro se destaca com contribuições no fortalecimento da qualidade dos sistemas de saúde, exercendo papel fundamental no reconhecimento das necessidades de cobertura e implementação da saúde, considerando as distintas realidades e necessidades vivenciadas pelas populações. ${ }^{2-3}$ No entanto, para exercer esta ação, faz-se necessário que os enfermeiros possuam conhecimentos específicos acerca das RAS, o que lhes permitirá reconhecer fragilidades e contribuir no processo de reorganização do modelo de atenção à saúde no SUS ${ }^{4}$.

Entendendo a relevância do papel desempenhado pelos enfermeiros na consolidação da RAS ${ }^{4}$, recai sobre este profissional a exigência de habilidades, competências e conhecimento específico. Neste sentido, a formação qualificada desempenha papel preponderante na qualificação profissional, para que atendam as demandas populacionais.

A formação do enfermeiro no Brasil segue as orientações das Diretrizes Curriculares Nacionais do Curso de Graduação em Enfermagem, publicadas em 2001, que buscam uniformizar o ensino e proporcioná-lo com a qualidade adequada, com a finalidade de estimular as instituições de ensino superior a adotarem métodos pedagógicos problematizadores, libertadores, e que proporcionem uma visão holística do ser humano e da sociedade ${ }^{5}$, atuando de modo a viabilizar a integralidade da atenção à saúde e o desenvolvimento do trabalho em equipe multiprofissional. Assim, a partir desse estudo, espera-se compreender o processo de formação de futuros enfermeiros para atuação nas RAS, servindo de sustentação para discussões sobre os Projetos Pedagógicos e incentivando novas investigações sobre o tema.

Considerando a vigência das Diretrizes Curriculares Nacionais do Curso de Graduação em Enfermagem, no ano de 2001, as intensas transformações ocorridas no âmbito do SUS deste mesmo ano até a atualidade, em especial no que se refere à estruturação das Redes de Atenção à Saúde, bem como a dinamicidade do processo formativo do enfermeiro no que se refere a sua capacitação para a atuação no SUS, este estudo apresenta a seguinte questão norteadora: qual a compreensão dos estudantes de graduação em enfermagem sobre as Redes de Atenção à Saúde? A partir dessa questão objetivou-se analisar a compreensão dos estudantes em enfermagem sobre da Rede de Atenção à Saúde.

\section{REFERENCIAL TEÓRICO}

As RAS constituem-se da combinação de elementos concentrados e dispersos articulados em pontos de atenção à saúde e em sistemas de apoio, que devem estar em equilíbrio para propiciar acesso aos serviços ${ }^{1}$. $O$ acesso é entendido como a capacidade do sistema de atenção à saúde de responder às necessidades de saúde apresentadas por uma determinada população, o que inclui a acessibilidade, a oferta, a territorialidade, dentre outros aspectos ${ }^{6}$.

Um dos principais autores sobre a temática refere que, para que haja uma estruturação eficiente, efetiva e de qualidade, deve-se seguir os seguintes fundamentos: economia de escala, disponibilidade de recursos, qualidade e acesso; integração horizontal e vertical; processos de substituição; territórios sanitários; e níveis de atenção ${ }^{7}$.

Sendo assim, além da quantidade e da qualidade dos serviços, é preciso oferecer acesso a população aos serviços de saúde. Nesta perspectiva, o Ministério da Saúde aponta que estratégias devem ser pensadas para possibilitar e garantir o acesso, principalmente, dos indivíduos com mais carências aos serviços e possíveis barreiras, de localização, culturais, sociais, de gênero e étnicas, devem ser desconstruídas ${ }^{8}$.

Destaca-se que a Atenção Primária em Saúde (APS), mesmo configurando-se como a principal porta de entrada ao sistema de saúde, não é considerada menos complexa em relação aos cuidados de média e alta complexidade, uma 
vez que esta é responsável por resolver mais de 85\% dos problemas de saúde e, que os níveis de atenção secundário e terciário apresentam maior densidade tecnológica, mas não de maior complexidade. Partindo desta compreensão, fazse necessária a substituição da concepção hierárquica pela concepção de redes poliárquicas de atenção à saúde, abandonando a ideia de organização do sistema e das relações de modo verticalizado, e adotando o entendimento de redes policêntricas horizontais ${ }^{1}$.

\section{MÉTOdo}

Estudo qualitativo, exploratório e descritivo, realizado com estudantes do nono e décimo semestres do Curso de Graduação em Enfermagem de uma universidade pública federal situada no sul do Brasil.

Foram incluídos no estudo todos os estudantes do Curso de Graduação em Enfermagem regularmente matriculados em, pelo menos, uma disciplina de estágio supervisionado e que cursaram todas as disciplinas teóricopráticas do curso. Os critérios de exclusão se limitaram à ausência do estudante no local e no momento de coleta de dados por falta, atestados ou licenças.

A coleta de dados foi realizada entre agosto e setembro de 2018, por meio de entrevista semiestruturada, individual e gravada, com duração média de 20 minutos. Depois de gravadas e transcritas, as falas dos estudantes foram identificadas pela letra $E$, de estudantes, seguida de um número contínuo (E1 a E27), como forma de garantir o anonimato.

Os dados foram submetidos à Análise Textual Discursiva que se fundamenta em um processo auto organizado de estruturação e compreensão, onde novas percepções surgem, por intermédio de uma sequência de três etapas: a desmontagem dos textos ou unitarização; o estabelecimento de relações ou categorização; e a captação do novo emergente ou comunicação ${ }^{9}$. A unitarização é o processo de fragmentação do material, resultando na exploração do texto com intuito de atingir as unidades de sentido. A categorização é a classificação em categorias, a partir do nexo entre as unidades base. Por fim, a comunicação é o resultado das duas etapas anteriores que revela a compreensão do todo ${ }^{9}$.

O estudo foi aprovado pelo Comitê de Ética em Pesquisa da instituição, com Parecer no 163/2018 e CAAE: 92504218.2.0000.5324. Todos os participantes assinaram o Termo de Consentimento Livre e Esclarecido.

\section{RESULTADOS E DISCUSSÃO}

Emergiram quatro categorias da análise textual discursiva dos dados: Entendimento sobre a RAS e suas contribuições para o SUS; Fatores que dificultam a efetivação das RAS; A atuação do enfermeiro na RAS; Formação acerca das RAS: Fragilidades e Propostas

\section{Entendimento sobre a RAS e suas contribuições para o SUS}

Para os estudantes, as RAS são os diferentes locais de assistência à saúde e se efetivam por meio da articulação entre esses diferentes serviços, como forma de organizar este processo, de modo a tornar o cuidado integral, contínuo e de qualidade. A percepção dos participantes no que tange o conceito das RAS vai em direção ao conceito proposto por Mendes ${ }^{1}$, conforme as falas a seguir:

É realmente uma rede, com estruturas que devem funcionar interligadas para o atendimento completo do indivíduo (E18).

As Redes de Atenção à Saúde são, como se fossem, o modo de operacionalizar o sistema único de Saúde para garantir a integralidade do acesso, para garantir que todos os setores estão sendo garantidos para o cidadão (E23).

As falas revelam, ainda, o conhecimento dos estudantes sobre a articulação da RAS, que se executa por meio de níveis de complexidade e propõem ações de promoção à saúde e prevenção e tratamento de doenças, o que também é descrito no modelo de Mendes ${ }^{1}$ e em outros estudos que abordam a temática ${ }^{10}$.

As Redes são, por exemplo, atenção primária, atenção secundária, atenção terciária. A primária são as UBSs, a secundária as UPAs e depois o complexo hospitalar é a terciária (E6).

É um sistema que oferta serviços de saúde de média, de baixa e de alta complexidade (E11).

Em relação às formas de acessar os serviços que compõe a RAS, os estudantes de enfermagem apontam para a influência do processo e organização através da hierarquização por complexidade, elencando a APS como principal porta de entrada. No entanto, o modelo das RAS aponta que é necessário substituir a ideia de organização do sistema e das relações de modo verticalizado, e adotar o entendimento de redes policêntricas horizontais ${ }^{7}$.

A porta [de entrada] é a Rede básica. A rede básica é a que fundamenta tudo, se ela for melhor implementada o hospital não teria esse fluxo tão grande (E17). 
É um atendimento público que deveria se iniciar pela atenção básica, pelo atendimento primordial que é dentro da comunidade e depois ser dividido para os outros níveis de atenção (E22).

Para os estudantes, a organização do sistema de saúde em um modelo de rede reduz a fragmentação do cuidado, sendo a dinamicidade do funcionamento da RAS o elemento efetivador dos princípios do SUS. Para efetivação do acesso e integralidade a saúde, se faz necessário planejar a disposição dos estabelecimentos de saúde, distribuídos em regiões de acordo com o nível de complexidade e da necessidade população em um determinado local ${ }^{1,11}$.

Contribui para a organização do sistema e para a continuidade do cuidado (E7).

Eu acho que contribui com a garantia do princípio da integralidade da assistência porque o indivíduo vai conseguir caminhar por todos os níveis de atenção, por todos os serviços. E vai, se ela funciona, se elas estão interligadas, ter as informações desse paciente correndo por toda a Rede (E11).

Também foi elencada pelos estudantes como potencialidade da RAS a aproximação de alguns serviços com as demandas de saúde das comunidades, em seus territórios, permitindo a atuação efetiva na dimensão dos cuidados de promoção da saúde e prevenção de doenças. A territorialização organiza o fluxo da RAS, estimulando a admissão do usuário no sistema de maneira correta, sem sobrecarregar apenas uma face do sistema ${ }^{12}$.

Gastaria menos dinheiro em coisas básicas. Poderia investir esse dinheiro mais na comunidade, em projetos que sanariam demandas daquela comunidade sem, depois, ter um grande gasto com os indivíduos nos hospitais (E2).

Acabaria com esse congestionamento de pacientes no hospital. Às vezes é uma coisa mínima que o paciente tem e acaba indo para o hospital, podendo ir à rede [básica], nos postos 24 horas ou numa UPA (E25).

Eu acho que diminuiria o elevado número de internações de pacientes (E26)

\section{Fatores que dificultam a efetivação das RAS}

Para os estudantes em enfermagem, as fragilidades na comunicação entre os serviços que compõe os níveis de atenção à saúde, faz com que haja a fragmentação ou a perda de informações, limitando a integração destes em prol do cuidado. Corroborando, um estudo realizado com trabalhadores da atenção primária e terciária de saúde mostrou que eles também identificam a falta de articulação e intercomunicação entre os serviços da RAS ${ }^{11}$ e em outro estudo revelou que trabalhadores da APS se sentem impossibilitados diante das restrições originadas por tais fragilidades ${ }^{13}$.

[...] Tem bastantes problemas, a função das Redes de Atenção não funciona porque o sistema não é interligado (E19).

Desse fluxo só vemos a doença, o paciente está doente, trata a doença e manda embora. Não vemos a continuação desse processo (E1).

Como forma de superar esse dificultador, são destacadas as Tecnologias de Informação e Comunicação (TIC), como o uso de sistemas informatizados para a partilha de dados referentes aos prontuários dos usuários do SUS. As TIC para a saúde favorecem a coparticipação do cuidado dos trabalhadores nos diferentes níveis de atenção garantindo integralidade e organização do atendimento ${ }^{14}$.

Se tivesse, principalmente, um sistema de informatização onde todas as áreas tivessem acesso ao prontuário do paciente e tivesse livre acesso da atenção básica, das UBS para o hospital (E18).

Também foi elencado como aspecto dificultador da efetivação da RAS, a falta de conhecimento da população sobre a estruturação da mesma e sobre como deveriam funcionar os fluxos de atendimento. Além das dificuldades de compreensão da população, problemas como a demora no agendamento de consultas via APS e o modelo de atenção à saúde voltado para o modelo biomédico dificultam ações de prevenção e promoção a saúde, fazendo com que os usuários procurem diretamente serviços especializados em busca de tratamento específico ${ }^{15}$.

Falta informação para o usuário, de que ele pode começar pelo nível mais básico e não chegar direto no hospital (E7).

Como instrumento para atenuar a fragilidade acima destacada, os estudantes de enfermagem remetem para a educação profissional acerca da legislação da RAS, bem como para uma maior participação dos profissionais na instrumentalização da população. A falta de conhecimento dos trabalhadores de saúde com relação as ações e serviços prestados $^{11}$ resulta num acolhimento despreparado e encaminhamento inadequado dos usuários, influenciando negativamente a prestação e continuidade do cuidado ${ }^{16}$.

O enfermeiro tem que conhecer bem a portaria [4.279 - criação das RAS] e saber articular com todos os programas, hospitais, a comunidade (E2).

Capacitar aquele enfermeiro, para ele saber, para ele orientar os pacientes da comunidade, da região dele, para eles saberem que, por exemplo: 'aqui a gente atende assim, dessa forma, e agora tu tens que te direcionar lá para o hospital'. Então, acho que falta capacitação para eles (E6). 


\section{A atuação do enfermeiro na RAS}

O enfermeiro é versado, pelos estudantes, como figura principal para o estabelecimento do vínculo com o usuário da rede, percebido como o profissional de referência para o cuidado, especialmente, na APS. Isso se dá devido à aproximação da APS com o usuário e a autonomia que o enfermeiro conquistou a partir de suas ações, indo para além da assistência, agindo de maneira multi e interdisciplinar ${ }^{17,18}$. No que tange o papel do enfermeiro na RAS, os estudantes demonstraram dificuldade em visualizar sua ação na pluralidade dos serviços propostos pela RAS. Nesse sentido, o papel do enfermeiro nas RAS é mais bem figurado quando abordado nas Redes Temáticas, que possuem intuito de atender as demandas de saúde da população².

O enfermeiro é a profissional chave, na verdade, em todos esses locais, tanto no hospital como numa rede básica (E1).

O enfermeiro é a porta de entrada pra atenção básica, para o cuidado de saúde acontecer. O enfermeiro é, principalmente na atenção básica, a referência para o cuidado, a referência para o vínculo, a referência para o paciente iniciar seu percurso na rede (E22).

Reforçando as falas anteriores, os estudantes concebem a atuação do enfermeiro na RAS como fundamental para que o cuidado a saúde aconteça. Destacam-se as ações de prevenção e promoção à saúde, ações que ocasionam um cuidado integral e uma maior resolutividade precedente a inevitabilidade de cuidados a nível terciário. Em um estudo realizado com estudantes da área da saúde, evidenciou-se a percepção sobre a vinculação do profissional com a comunidade, sendo ponto principal no êxito para o cuidado integral ${ }^{19}$.

O enfermeiro tem que ter um olhar que consiga captar algo que ele possa resolver antes de chegar numa internação hospitalar (E5).

Eu vejo que o enfermeiro é um dos principais profissionais no contato direto com o paciente. Tanto na prevenção quanto no tratamento, ele tem a visão do que realmente o paciente precisa (E23).

Ainda que, através das falas, os participantes tenham expressado um insuficiente aprofundamento no que concerne à atuação do enfermeiro na gestão da RAS, foi apontada a importância da atuação do enfermeiro para a organização e funcionamento da mesma para que haja a efetivação do que é proposto pela RAS. O enfermeiro se destaca a partir de ações de prevenção, de promoção, de educação, administrativas e gerenciais ${ }^{17}$, atributos necessários para consolidação e efetivação da RAS.

O enfermeiro contribui para rede facilitando a comunicação entre os níveis de assistência e também com a secretaria de saúde (E7).

O enfermeiro precisa conhecer a Rede, conhecer como ela se estrutura, como se dá sua dinâmica, seus fluxos. Porque eu acho que a gente tem a responsabilidade de garantir seu funcionamento. [...] Tem a responsabilidade de orientar também os pacientes e os próprios profissionais sobre o funcionamento (E11).

\section{Formação acerca das RAS: Fragilidades e Propostas}

As falas permitem evidenciar que há grande ênfase do ensino para a assistência de enfermagem na área hospitalar, tendo pouco destaque na formação os demais serviços que compõem as RAS. Tal contexto acaba por proporcionar uma exposição insuficiente dos estudantes à perspectiva de formação da rede, levando a aquisição de um conhecimento fragmentado em torno da gestão/funcionamento. Logo, aparentemente, a formação do enfermeiro ainda pouco o estimula a refletir sobre seu papel enquanto agente de saúde, sendo sua formação pautada na aprendizagem e desenvolvimento de ações tecnicistas ${ }^{20}$.

Visualizo muito voltado para a alta complexidade, a nossa formação é muito voltada para o hospital e a gente vê pouca unidade básica, vamos descobrir mesmo agora no décimo [semestre]. Com relação aos setores de vigilância em saúde, aos setores mais administrativos, que não envolve tanta assistência direta ao paciente é bem superficial (E7).

Os estudantes revelam que sua formação não permite uma visão ampliada dos serviços enquanto integrantes da RAS. Além disso, apontam para um fazer acadêmico na APS que difere da atuação do enfermeiro neste ambiente. Tal perspectiva faz com que não se sintam suficientemente instrumentalizados para atuarem na APS no prisma de efetivação das RAS quando terminarem seu processo de formação. A formação deve ofertar subsídios para emergir um perfil de enfermeiro generalista, humanista, questionador e reflexivo, que defenda os princípios e diretrizes do SUS e, sobretudo, assegure a assistência em sua integralidade ${ }^{21}$.

[...] a gente passa pela unidade básica e, nesse tempo, a gente não faz o que o enfermeiro faz, a gente vê pouco isso (E27).

[...] se eu me formar e for trabalhar lá na unidade [básica de saúde] eu não vou saber o que eu tenho que fazer, como que encaminho ele, qual a orientação que eu dou (E16). 
Diante do exposto, para superar a fragmentação destacada, fortalecer o ensino e preparar os estudantes para atuarem na RAS, o processo de formação deve fomentar a discussão sobre a RAS entre as disciplinas ofertadas e a transversalidade entre o ensino e a prática. Nesse contexto, os novos modelos de atenção à saúde devem apoiar e direcionar a formação dos enfermeiros capazes de empregar o conhecimento de forma interdisciplinar, orientado para o cuidado integral em todos os níveis de atenção à saúde $22,23$.

Temos uma disciplina, talvez duas, que falam sobre isso, deveria ser transdisciplinar, deveria falar em todas, pelo menos alguma coisa sobre isso [sobre as RAS] (E3).

Tivemos a disciplina do SUS, para identificar e estudar, muito no início da graduação. Deveria ao longo da graduação continuar pra gente se adaptar e entender melhor (E24).

A limitação deste estudo refere-se à sua realização com uma amostra de estudantes de uma única instituição de ensino, fator não propício à generalização dos resultados.

\section{CONCLUSÃO E IMPLICAÇÕES PARA A PRÁTICA}

Os estudantes apresentaram conhecimento parcial acerca da RAS, com dificuldade na compreensão quanto ao modelo de organização proposto pela RAS, pautado em redes policêntricas horizontais, não hierarquizadas. Apesar disso, apresentaram sabedoria sobre alguns pontos chaves como o conceito e o objetivo da RAS; clareza sobre a articulação feita entre os níveis de atenção à saúde, identificando a APS como porta de entrada para o sistema. Ademais, conseguiram perceber fragilidades na estrutura da RAS, que resultam na desintegração do sistema e na fragmentação do cuidado, fragilizando a execução da RAS enquanto efetivadora do SUS.

A percepção dos estudantes em relação à falta de conhecimento sobre a atuação do enfermeiro nos diversos serviços da RAS é consequência da formação fragmentada, que prioriza o ensino pautado em ações curativas e que não aborda, de forma efetiva, a inserção dos estudantes no sistema, bem como da falta de DCN para enfermagem que estejam consoantes com as propostas das RAS. Tais fatores enfraquecem o aprendizado teórico-prático nos diferentes níveis de atenção, resultando na geração de profissionais insuficientemente preparados para atuarem como agentes colaboradores da concretização da RAS.

O estudo possibilitou a visualização de como o processo de formação do estudante de enfermagem os capacita sobre as Redes de Atenção à Saúde. Os achados podem servir para que docentes e discentes reflitam sobre o estabelecimento de Projetos Pedagógicos que sanem as demandas educacionais e sejam capazes de contribuir para a efetivação do SUS e das RAS.

\section{REFERÊNCIAS}

1. Mendes EV. As redes de atenção à saúde. Brasília (DF): Organização Pan-Americana da Saúde; 2011

2. Conselho Nacional de Secretários de Saúde (BR). A Atenção Primária e as Redes de Atenção à Saúde. Brasília (DF): CONASS; 2015.

3. Organização Pan-Americana da Saúde (OPAS). A atenção à saúde coordenada pela APS: construindo as redes de atenção no SUS. Brasília (DF): Organização Pan-Americana da Saúde; 2011.

4. Moll MF, Goulart MB, Caprio AP, Ventura CAA, Ogoshi AACM. The knowledge of nurses on health care networks. Rev. enferm. UFPE online [internet]. 2017 [cited 2019 Nov 25]; 11(1):86-93. Available from: https://periodicos.ufpe.br/revistas/revistaenfermagem/article/view/11881

5. Brito MCC, Teófilo FKS, Dias MAS, Vasconcelos MIO, Albuquerque IMN, Silva LCC. Training of nurses for primary health care: a look at the knowledge produced. Sanare [internet]. 2017 [cited 2019 Nov 25]; 16(2):93-102. Available from: https://sanare.emnuvens.com.br/sanare/article/viewFile/1183/644

6. Donabedian A. Aspects of Medical Care Administration: Specifying Requirements for Health Care. Cambridge MA: Harvard University Press; 1973.

7. Mendes EV. Health care networks. Ciênc. saúde coletiva [Internet]. 2010 [cited 2019 Nov 25]; 15(5):2297-305. DOI: https://doi.org/10.1590/S1413-81232010000500005

8. Ministério da Saúde (BR). Portaria GM/MS no 4.279, de 30 de dezembro de 2010. Estabelece as diretrizes para a organização da Rede de Atenção a Saúde no âmbito do SUS. Brasília (DF): Ministério da Saúde; 2010 [cited 2019 Nov 25]. Available from: https://bvsms.saude.gov.br/bvs/saudelegis/gm/2010/prt4279_30_12_2010.html

9. Moraes R, Galiazzi MC. Análise Textual Discursiva. 3ạ ed. Ijuí (SC): Unijuí; 2016.

10. Kalichman AO, Ayres JRCM. Comprehensiveness and healthcare technologies: a narrative on conceptual contributions to the construction of the comprehensiveness principle in the Brazilian Unified National Health System. Cad. Saúde Pública [Internet]. 2016 [cited 2020 Jul 11]; 32(8):e00183415. DOI: https://doi.org/10.1590/0102-311X00183415

11. Brondani JE, Leal FZ, Potter C, Silva RM, Noal HC, Perrando MS. Challenges of referral and counter-referral in health care in the workers' perspective. Cogitare Enferm. [internet]. 2016 [cited 2019 Nov 25]; 21(1):1-8. Available from:

https://revistas.ufpr.br/cogitare/article/view/43350/27700 
12. Faria R. Territorialization of primary health care in sus: evaluation of results in a city in the southern region of brazil abstract. Hygeia [Internet]. 2018 [cited 2019 Nov 25]; 14(29):89-104. DOI: https://doi.org/10.14393/Hygeia142908

13. Macedo LM, Martin STR. Interdependence between the levels of care in Brazilian Health Sistem (SUS): meanings of integrality reported by primary care workers. Interface (Botucatu) [Internet]. 2014 [cited 2020 Jul 11]; 18(51):647-60. DOI: https://doi.org/10.1590/1807-57622013.0597

14. Magalhães Junior HM. Health Care Networks: towards the integrality. Divulg. saúde debate [Internet]. 2014 [cited 2020 Jul 11]; 52:15-37. Available from: http://cebes.org.br/site/wp-content/uploads/2014/12/Divulgacao-52.pdf

15. Silva TR, Motta RF. Users' perceptions of health policy in primary care. Mudanças [Internet]. 2015 [cited 2020 Jul 11]; 23(2):1725. DOI: https://doi.org/10.15603/2176-1019/mud.v23n2p17-25

16. Quevedo ALA, Rossoni E, Pilotto LM, Pedroso MRO, Pacheco PM. Direito à saúde, acesso e integralidade: análise a partir de uma unidade saúde da família. Rev. APS [internet]. 2016 [cited 2019 Nov 25]; 19(1):47-57. Available from: https://periodicos.ufjf.br/index.php/aps/article/view/15640

17. Freitas GM, Santos NSS. Performance of the nurse in primary health: integrative review of literature. Rev. Enferm. Cent. O. Min. [Internet]. 2014 [cited 2019 Nov 25]; 4(2):1194-203. Available from: http://www.seer.ufsj.edu.br/index.php/recom/article/view/443/754

18. Ferreira GE, Santos EP, Gomes ASD, Capellari C, Morais RTR. Community interventions in nursing: intersectoriality, entrepreneurship training and regional health development. Redes [Internet]. 2018 [cited 2020 Jul 11]; 23(3):317-35. DOI: http://dx.doi.org/10.17058/redes.v23i3.12182

19. Gomes TLCS, Higa EFR, Passos AHR, Soares MOM, Otani MAP, Souto BGA. Comprehensiveness in the primary health care system: understanding of medical and nursing students. Rev. Psi. Divers. Saúde [Internet]. 2018 [cited 2019 Nov 25]; 7(01):1129. DOI: http://dx.doi.org/10.17267/2317-3394rpds.v7i1.1898

20. Alberti GF, Schimith MD, Budó MLD, Neves GL, Rosso LF. First-contact accessibility attribute in primary health care services and care practices: contributions to academic education for nurses. Texto contexto - enferm. [Internet]. 2016 [cited 2020 Jul 11]; 25(3):e4400014. DOI: https://doi.org/10.1590/0104-07072016004400014

21. Corrêa AB, Reibnitz KS, Kloh D, Prado ML, Rodrigues J, Lima MM. Contributions of the pro-health program: a vision of nursing exchange. Rev. enferm. UFPE online [Internet]. 2017 [cited 2019 Nov 25]; 11(2):567-75. Available from: https://periodicos.ufpe.br/revistas/revistaenfermagem/article/view/11975/14524

22. Winters JRF, Prado MR, Heidemann ITSB. Nursing education oriented to the principles of the Unified Health System: perception of graduates. Esc. Anna Nery [Internet]. 2016 [cited 2019 Nov 25]; 20(2):248-53. Available from: https://www.scielo.br/scielo.php?script=sci_arttext\&pid=S1414-81452016000200248\&lng=en\&tIng=en

23. Brehmer LCF, Ramos FRS. The healthcare model in training for nursing: experiences and perceptions. Interface (Botucatu) [Internet]. 2016 [cited 2019 Nov 25]; 20(56):135-45. DOI: https://doi.org/10.1590/1807-57622015.0218 